\title{
Beam Characterization for Scanning Electron Microscopes by the RPS and IPC Methods
}

\author{
Tomoyo Sasaki ${ }^{1,2}$, Mitsugu Sato ${ }^{1}$, Kishio Hidaka ${ }^{3}$ and Jin Onuki ${ }^{2}$ \\ ${ }^{1 .}$ Hitachi High-Technologies Corporation, 882, Ichige, Hitachinaka-shi, Ibaraki-ken, 312-8504, Japan. \\ ${ }^{2}$ Graduate School of Science and Engineering, Ibaraki University, 4-12-1, Nakanarusawa-cho, \\ Hitachi-shi, Ibaraki-ken, 316-8511, Japan. \\ ${ }^{3}$ Department of Education, Art and Science, Yamagata University,1-4-12, Kojirakawamachi, \\ Yamagata-shi, Yamagata-ken, 990-8560, Japan.
}

The spatial resolution of scanning electron microscopes (SEMs) is strongly related to the electron-beam current intensity profile, which in turn has related characteristic size measures. Other factors influencing the spatial resolution include details of the observed sample and the noise in the system. However, including all such relevant factors is often impractical when seeking an optimized electron optical system design and thus resolution measure estimates are often employed, based purely on simple theoretical considerations [1]. In this case, one should use the most appropriate theoretical resolution measure that will ideally give the best resolution measure in the realized SEM system.

In many cases with SEMs, the beam intensity profile is often approximately Gaussian and thus the theoretical resolution defined by the full width-half maximum (FWHM) of the beam intensity profile is normally used and valid enough. However, the FWHM of the beam is not suitable to estimate the theoretical resolution of non-Gaussian beams. For example, when increasing the beam-aperture angle and adjusting the defocus position, the FWHM of the beam can be kept small but the beam tail generated by spherical aberration becomes apparent and degrades image quality [2]. Moreover, in low-current and high-resolution imaging applications of an SEM with a field-emission (FE) electron source, the beam intensity profile is often better described by an Airy pattern, rather than a Gaussian profile, owing to the wave properties of the electrons being dominant in this situation [3].

A common method to calculate the beam size is to take the square-root of the sum of the squared measures of the major beam-intensity profile contributors: the electron-source size referred to the image plane; and size measures related to diffraction, spherical and chromatic aberrations [4]. This method is widely used to estimate the theoretical resolution of SEMs but the accuracy of calculation is not high because the beam intensity profile is not taken account of exactly. As more high-precision methods, the root-power-sum (RPS) method and the information-passing-capacity (IPC) method have been suggested $[5,6]$. These methods take account of the beam intensity profile but are based on different concepts. Therefore, one needs to understand the physical meanings and the common or different features of these methods to use them appropriately in a given situation.

In this paper, we demonstrate the properties of the RPS and IPC methods by comparing their resolution measures with more exactly modeled beam intensity profiles and brightness-contrast characteristics of particles and lines in anticipation of low-current and high-resolution imaging applications.

The RPS method is based on the concept of the fraction of current $F C$ [5]. The RPS resolution $R_{\mathrm{RPS}}$ corresponds with the beam diameter containing a given $F C$ to the total beam current. As shown in Figure 1, when integrating a beam intensity profile over the inside of a circle having a certain diameter $d$, 
the ratio of the integrated value to the total current is the $F C$. The behavior of the $F C$ s of the Airy pattern and the Gaussian profile as a function of an integrated-circle diameter $d$ is also shown in Figure 1. With reference to the Airy pattern in Figure 1, the RPS resolution $R_{\mathrm{RPS}}$ of an $F C$ of $75 \%$ is $4.5 \mathrm{~nm}$ and the RPS resolution $R_{\mathrm{RPS}}$ of an $F C$ of $25 \%$ is $2 \mathrm{~nm}$. Therefore, the RPS resolution $R_{\mathrm{RPS}}$ greatly depends on the choice of an $F C$. Barth and Kruit have said that how to choose an $F C$ for each application could be investigated by taking account of the optical transfer function of the beam intensity profile [5]. We would like to contribute to the investigation by comparing the RPS resolution $R_{\mathrm{RPS}}$ with the IPC resolution $R_{\mathrm{IPC}}$ derived from the optical transfer function in this paper.

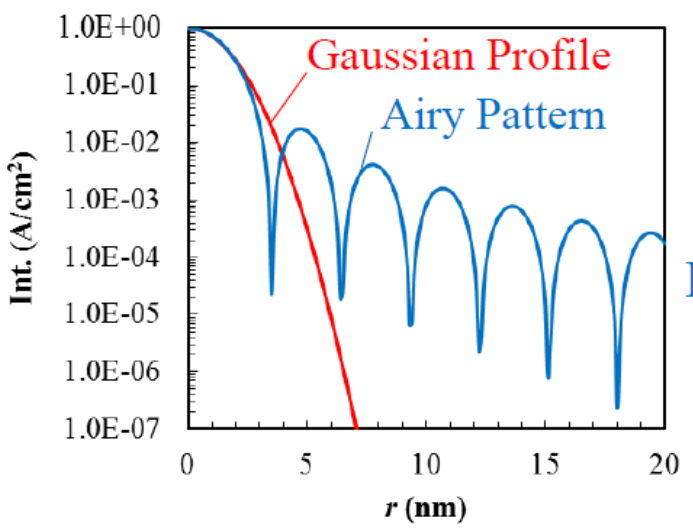

Beam Intensity Profiles (BIPs)

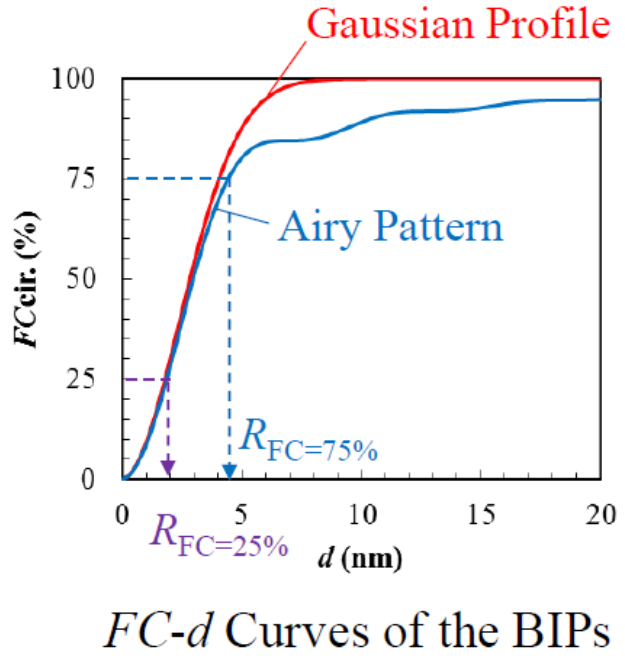

Figure 1. The concept of the root-power-sum (RPS) method: the beam intensity profiles of the Airy pattern and the Gaussian profile, and the fraction of current $F C$ to an integrated-circle diameter $d$ curves obtained by the integration of the beam intensity profiles.

The algebraic formula for calculating the RPS resolution $R_{\mathrm{RPS}}$ of the entire $F C$ has been suggested in the RPS method, where this formula has been determined by fitting the RPS resolution $R_{\mathrm{RPS}}$ to the exact results simulated from the beam intensity profiles [5]. When using the formula, the RPS resolution $R_{\mathrm{RPS}}$ is calculated at the optimum defocus position $\Delta z_{\mathrm{RPS}}$ leading the best RPS resolution $R_{\mathrm{RPS}}$ of the optical-axis positions, where the optimum defocus position $\Delta z_{\mathrm{RPS}}$ is calculated as the defocus position $\Delta z_{\mathrm{DLC}}$ of the disc of least confusion multiplied by an $F C$.

The IPC method is based on the concept of information content [6]. Generally, in the case of digital images, the information content $H$ (bit) is calculated by $H=M \log _{2} m$, where the uppercase $M$ equates to the number of pixels and the lowercase $m$ equates to the number of brightness gradations. On the other hand, in the case of analogue images, blur decreases the effective number of pixels and brightness noise decreases the effective number of brightness gradations so the information content of analogue images is determined by blur and brightness signal-to-noise ratio $S N R$. Linfoot has quantified this concept and suggested a formula for calculating the information-content density $\rho$ of analogue images [7]. In the case of SEM images, blur is produced by the finite beam size and sample-dependent extent of the signal-generation region, and the brightness SNR depends on the dose of electron beam along with the signal-electron yield of sample, amongst other factors. Sato and Orloff have applied the Linfoot formula to the definition of the theoretical resolution of SEMs, which is the IPC method [6]. 
According to the IPC method, the information-content density $\rho$ of SEM images can be obtained by

$$
\rho=\frac{1}{\ln 2} \iint \ln \sqrt{1+\left|f_{\text {OTF }} f_{S} f_{O} f_{A}\right|^{2} \cdot \frac{S}{N}} d v_{x} d v_{y}
$$

where $f_{\mathrm{OTF}}, f_{\mathrm{S}}, f_{\mathrm{O}}$, and $f_{\mathrm{A}}$, which are functions of the two dimensional spatial frequencies $v_{\mathrm{x}}, v_{\mathrm{y}}$, are the optical transfer function of the beam intensity profile of the electron optical system for a point source (the "point-spread function"), the Fourier transform of the source current distribution referred to the image plane, the Fourier transform of the sample structure including the blur of the signal-generation region on sample, and the acceptance factors determined by the pixel size of the image, respectively [8]. In addition, $S$ and $N$ are the statistical mean power densities of the signal and of the noise, respectively so $S / N$ indicates the signal-to-noise ratio $S N R$. In the IPC method, a sample is assumed as ideal for the evaluation of the theoretical resolution, i.e., an "infinite information" source $\left(\left|f_{\mathrm{O}}\right|=1\right)$, the information-content density $\rho$ in the formula (1) can be defined as a performance of an SEM for imaging, that is the information-passing capacity (IPC) under a given $S N R$.

Finally, based on the Airy radius $R_{\text {Airy }}$ of the Airy pattern, the IPC resolution $R_{\mathrm{IPC}}$ is calculated from the IPC value $\rho$ of an SEM by

$$
R_{\mathrm{IPC}}=R_{\text {Airy }}\left(\rho_{\text {ideal }} / \rho\right)^{1 / 2},
$$

where $\rho_{\text {ideal }}$ equates to the IPC value determined by only the optical transfer function $f_{\text {OTF }}$ of the Airy pattern $\left(\left|f_{S} f_{\mathrm{o}} f_{\mathrm{A}}\right|=1\right)$ under the same given $S N R$ as the formula (1). The formula (2) means that the IPC resolution $R_{\mathrm{IPC}}$ agrees with the Airy radius $R_{\text {Airy }}$ when the beam intensity profile is the Airy pattern. The Airy radius $R_{\text {Airy }}$ is defined as the spatial resolution of optical instruments in the Rayleigh criterion [9]. Therefore, the IPC resolution $R_{\mathrm{IPC}}$ is based on the concept of not only information content but also of the Rayleigh criterion.

The relationship between the RPS and IPC resolutions $R_{\mathrm{RPS}}, R_{\mathrm{IPC}}$ and the beam aperture half angle $\alpha$ is shown in Figure 2a. Here, diffraction and spherical aberration are considered as the factors deciding the optical transfer functions $f_{\text {OTF }}$ of the point-spread function in the Eq. (1), where the wave optical simulation is used to derive the functions $f_{\mathrm{OTF}}$. In addition, the source size referred to the image plane is assumed as infinitesimal, as in earlier work [2]. Therefore, the subsequent results correspond best with low-current and high-resolution imaging applications of a high-energy electron beam produced from a cold FE source. Investigation of high-current or low-energy regime will be carried out in the future. In Figures $2 \mathrm{a}$ and $2 \mathrm{~b}$, the energy $V$ of the electron beam is $5 \mathrm{keV}$ and the spherical aberration coefficient $C_{\mathrm{s}}$ of the lens is $3.1 \mathrm{~mm}$. In this paper, the optimum beam angle $\alpha_{\mathrm{opt}}$ is defined as the beam angle $\alpha$ leading to the best resolution of each $R$ - $\alpha$ curve. Moreover, the diffraction area is defined as the left-side area of the optimum beam angle $\alpha_{\text {opt }}$ because diffraction predominantly determines the beam intensity profile, where the beam intensity profile becomes the Airy pattern. On the other hand, the spherical aberration area is defined as the right-side area of the optimum beam angle $\alpha_{\text {opt }}$ because spherical aberration predominantly determines the beam intensity profile, where the beam intensity profile differs from the Airy pattern. In addition, the optimum defocus positions $\Delta z_{\mathrm{RPS}}, \Delta z_{\mathrm{IPC}}$, which is the optical-axis position leading to the best resolution following each method, of the RPS and IPC methods are shown in Figure 2b. Here, $\Delta z=0 \mathrm{~nm}$ indicates the optical-axis position of the Gaussian image plane. 
As shown in Figure 2a, the larger the $F C$ becomes, the worse the RPS resolution $R_{\mathrm{RPS}}$ becomes. Moreover, not only the RPS resolution $R_{\mathrm{RPS}}$, but also the optimum beam angle $\alpha_{\text {opt }}$ of the RPS method of each $F C$ changes with the choice of an $F C$. So it is important to understand how to choose an $F C$.

(a)

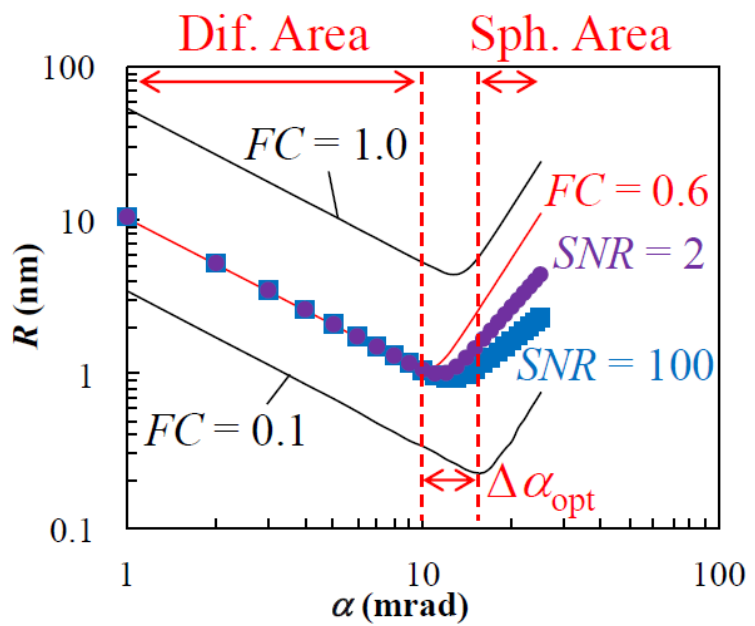

(b)

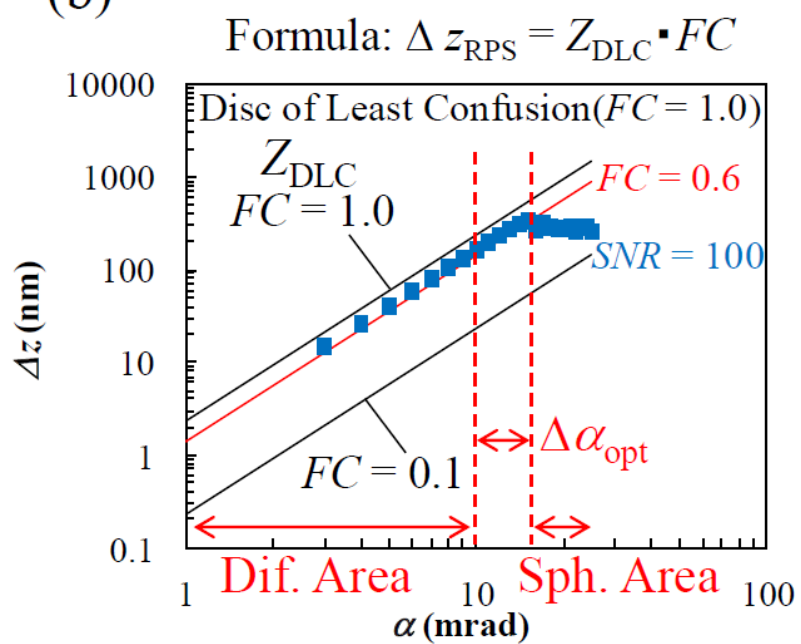

Figure 2. (a) The relationship between the RPS and IPC resolutions $R_{\mathrm{RPS}}, R_{\mathrm{IPC}}$ and the beam aperture half angle $\alpha$, where the solid lines indicate the RPS resolutions $R_{\mathrm{RPS}}$ and the circles and squares indicate the IPC resolutions $R_{\mathrm{IPC}}$. (b) The relationship between the optimum defocus positions $\Delta z_{\mathrm{RPS}}, \Delta z_{\mathrm{IPC}}$ of the RPS and IPC methods and the beam aperture half angle $\alpha$, where the solid lines indicate the optimum defocus positions $\Delta z_{\text {RPS }}$ of the RPS method and the squares indicate the optimum defocus positions $\Delta z_{\text {IPC }}$ of the IPC method of an SNR of 100.

In the diffraction area, the IPC resolution $R_{\mathrm{IPC}}$, which agrees with Airy radius $R_{\text {Airy }}$, and the RPS resolution $R_{\mathrm{RPS}}$ of an $F C$ of $60 \%$ are in agreement. These optimum defocus positions $\Delta z_{\mathrm{RPS}}, \Delta z_{\mathrm{IPC}}$ are also nearly aligned, which means that the calculations of both methods are carried out on the Airy pattern at the same optical-axis position. This result indicates that the RPS resolution $R_{\mathrm{RPS}}$ of an $F C$ of $60 \%$ of the Airy pattern is equivalent to the Airy radius, which is the spatial resolution defined by the Rayleigh criterion.

Further, the RPS resolution $R_{\mathrm{RPS}}$ of an $F C$ of $60 \%$ of the Gaussian profile corresponds with the effective diameter of the Gaussian profile, which is defined as the diameter where the beam intensity decreases to $1 / e$ of its maximum. The effective diameter of the Gaussian profile is defined as the theoretical resolution of the Gaussian profile by approximately using the Rayleigh criterion [2]. Therefore, the RPS resolution $R_{\mathrm{RPS}}$ of an $F C$ of $60 \%$ is valid for the estimation of the theoretical resolution for not only the Airy pattern but also the Gaussian profile. Furthermore, and importantly for SEM beam characterization, both the optimum beam angle $\alpha_{\mathrm{opt}}(=10 \mathrm{mrad})$ and the resolution $R_{\mathrm{RPS}}(=1.07 \mathrm{~nm})$ at the optimum beam angle $\alpha_{\text {opt }}$ of the RPS method with an $F C$ of $60 \%$ are almost in agreement with the values $\alpha_{\text {opt }}(=12$ $\mathrm{mrad}), R_{\mathrm{IPC}}(=0.98 \mathrm{~nm})$ of the IPC method, respectively. From now on, the $F C$ of $60 \%$ is used.

However, when it comes to the spherical aberration area, the RPS resolution $R_{\text {RPS }}$ of an $F C$ of $60 \%$ and the IPC resolutions $R_{\mathrm{IPC}}$ are different. In this area, the optimum defocus positions $\Delta z_{\mathrm{RPS}}, \Delta z_{\mathrm{IPC}}$ of the RPS and IPC methods also differ. Because the beam intensity profile with spherical aberration changes greatly depending on the optical-axis position, the optimum defocus position $\Delta z$ of each method should be taken account when discussing the properties of these methods. According to the definitions of the 
RPS and IPC resolutions, the average current density in a circle having a diameter of the RPS resolution $R_{\mathrm{RPS}}$ is maximum at the RPS-optimum defocus position $\Delta z_{\mathrm{RPS}}$, and the axial (central) current density of the beam intensity profile is maximum at the IPC-optimum defocus position $\Delta z_{\text {IPC}}$; these defocus positions are going away from each other in the spherical aberration area [2]. In Figure 2b, the optimum defocus position $\Delta z_{\text {IPC }}$ of the IPC method with an $S N R$ of 100 is only shown because it is approximately constant between an SNR of 100 and 2.

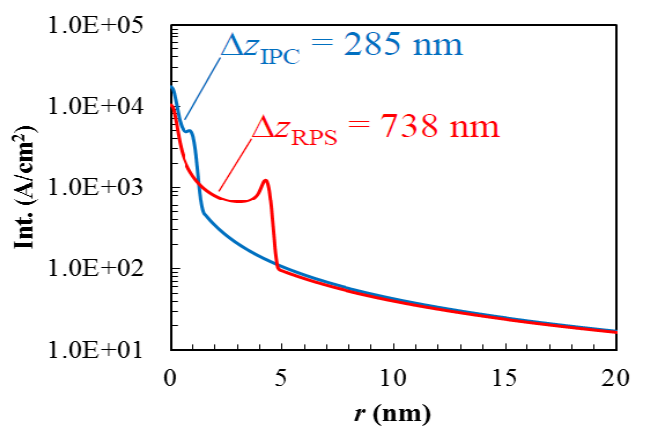

Beam Intensity Profiles (BIPs)

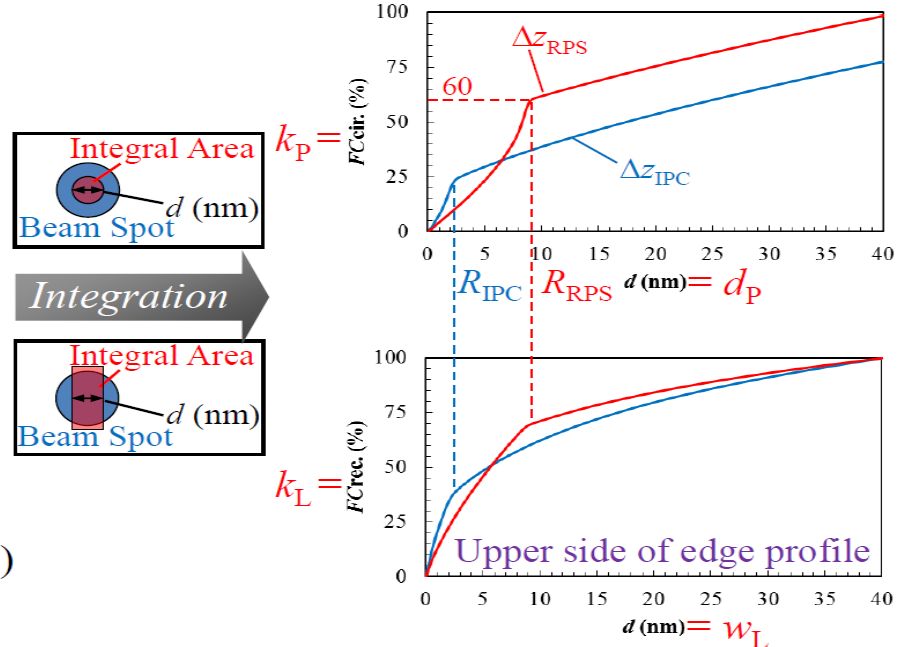

$F C$ - $d$ Curves of the BIPs

Figure 3. The beam intensity profiles simulated at the optimum defocus positions $\Delta z_{\mathrm{RPS}}(=738 \mathrm{~nm})$, $\Delta z_{\mathrm{IPC}}(=285 \mathrm{~nm})$ of the RPS and IPC methods in the spherical aberration area $(\alpha=23 \mathrm{mrad})$, and the fraction of current $F C$ to an integration diameter/width $d$ curves obtained by the integration of the beam intensity profiles.

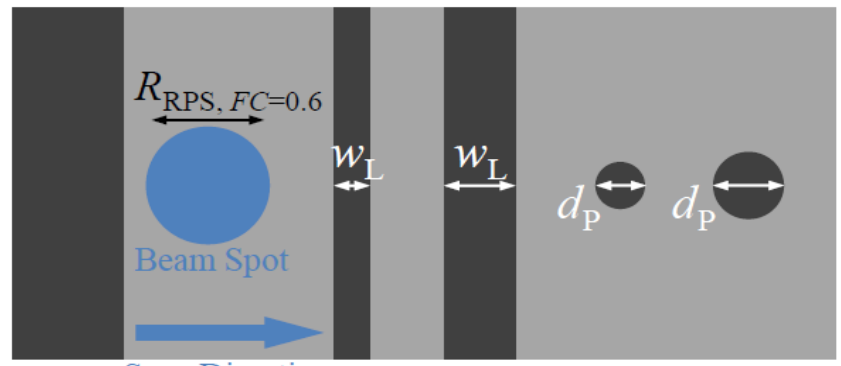

Scan Direction

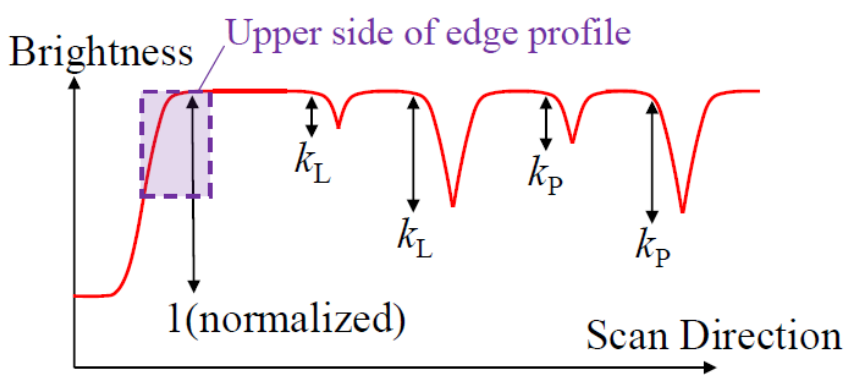

Figure 4. The ideal sample structure having the binary step contrasts of lines and particles, and the brightness contrasts of particles/lines and the edge profile of the ideal sample.

By using the ray trace simulation, the beam intensity profiles are simulated at the optimum defocus positions $\Delta z_{\mathrm{RPS}}(=738 \mathrm{~nm}), \Delta z_{\mathrm{IPC}}(=285 \mathrm{~nm})$ of the RPS and IPC methods in the spherical aberration area ( $\alpha=23 \mathrm{mrad}$ ) [2]. In this simulation, instead of the Airy pattern having the Airy radius of $0.044 \mathrm{~nm}$ of diffraction, the equivalent Gaussian profile having the effective diameter of $0.044 \mathrm{~nm}$ is approximately used, where "equivalent" means the same theoretical resolutions of $0.044 \mathrm{~nm}$. By integrating these beam intensity profiles, the fraction of current $F C$ to an integration diameter/width $d$ curves are obtained, as shown in Figure 3. Here, not only the circular integration but also the rectangular integration are carried 
out. This simulated RPS resolution $(9.0 \mathrm{~nm})$ almost agrees with the calculated value $(8.6 \mathrm{~nm})$ of the RPS method. From Figure 3, it can be seen that the RPS and IPC resolutions $R_{\mathrm{RPS}}, R_{\mathrm{IPC}}$ should indicate the beam diameter of the break point on each $F C$ to $d$ curve. With reference to the rectangular integration, the diameters of the break points are in agreement with the values of the circular integration, and the curve disagreement between the RPS and IPC defocus potions is smaller than the case of the circular integration.

By assuming an ideal sample having the binary step contrasts shown in Figure 4, the brightness contrasts of particles/lines and the edge profile can be estimated from the $F C$ to $d$ curves, as shown in Figures 3 and 4. When the brightness contrasts of the edge, lines, and particles are normalized by the edge contrast, the $F C_{\text {cir. }}$ of the circular integration corresponds with the brightness contrast of particles $k_{\mathrm{p}}$. Moreover, the $F C_{\text {rec. }}$ of the rectangular integration corresponds with the brightness contrast of lines $k_{\mathrm{L}}$ and with the upper side of the edge profile. Based on this concept, the physical meaning of the $F C$ to $d$ curve is similar to the optical transfer function. As shown in Figures 3 and 4, it can be seen that the brightness contrast, or detection sensitivity, for smaller particles $\left(d_{\mathrm{p}}<6.5 \mathrm{~nm}\right)$ is better at the IPC defocus position $\Delta z_{\mathrm{IPC}}$. On the other hand, the brightness contrast for larger particles $\left(d_{\mathrm{p}}>6.5 \mathrm{~nm}\right)$ is better at the RPS defocus position $\Delta z_{\text {RPS }}$. With regard to the contrasts of lines and the edge profile, the discrepancy between the RPS and IPC method is relatively small. By using the beam at the IPC defocus position $\Delta z_{\mathrm{IPC}}$, higher gradient of the edge profile could be obtained but the contrast of the high-gradient part is small and thus users should take into account of whether the high-gradient contrast is enough for application.

In this paper, we have demonstrated the properties of the RPS and IPC methods by comparing their resolution measures with more exactly modeled beam intensity profiles and brightness-contrast characteristics of particles and lines, in order to appropriately use the RPS and IPC methods in low-current and high-resolution imaging applications. When the beam intensity profile is the Airy pattern or the Gaussian profile, the RPS resolution of the fraction of current $F C$ of $60 \%$ and the IPC resolution almost agree with the theoretical resolution defined by the Rayleigh criterion. When the beam intensity profile differs from the Airy pattern or the Gaussian profile by aberration, the RPS resolution of an $F C$ of $60 \%$ and the IPC resolutions are different. The RPS method of the $F C$ of $60 \%$ and the IPC method derive the defocus positions of the beam profiles suitable for the larger-particle contrast and the smaller-particle contrast, respectively. However, with reference to the contrast of lines and the edge profiles, the difference between the methods is relatively small. In the case of the non-Airy patterns, the beam characterization method should be chosen while taking account of sample-structure size and shape [10].

\section{References:}

[1] M Sato in "Handbook of Charged Particle Optics", ed. J Orloff, (CRC, New York) p. 391.

[2] M Sato, Nucl. Instrum. Meth. A 645 (2011), p. 74.

[3] AV Crewe, Ultramicroscopy 23 (1987), p. 159.

[4] L Reimer in "Scanning Electron Microscopy", (Springer, Berlin) p. 32.

[5] JE Barth and P Kruit, Optik 101 (1996), p. 101.

[6] M Sato and J Orloff, Ultramicroscopy 41 (1992), p. 181.

[7] EH Linfoot, J. Opt. Soc. Am. 45 (1955), p. 808.

[8] M Sato et al, Nucl. Instrum. Meth. A 519 (2004), p. 280.

[9] L Rayleigh, Phil. Mag. 8 (1879), p. 261.

[10] The authors are grateful to Dr. A. M. Blackburn for his valuable comments to this work. 